\title{
DIAGNÓSTICO DE ENFERMAGEM DE PACIENTES EM PÓS-OPERATÓRIO DE TRANSPLANTE HEPÁTICO POR CIRROSE ETÍLICA E NÃO-ETÍLICA
}

\author{
Nursing Diagnosis of Post Surgical Patients of Liver Transplantation \\ by Alcoholic and Non Alcoholic Cirrhosis \\ Diagnósticos de Enfermería de Pacientes en Pos Operatorio \\ de Transplante Hepático por Cirrosis Etílica y No Etílica
}

Daclé Vilma Carvalho ${ }^{1}$

Roberta Azevedo Carneiro 3
Márcia Eller Miranda Salviano²

Fabrícia Madalena Meira Santos ${ }^{4}$

\section{Resumo}

Os transplantes em algumas áreas passaram a ser opção curativa, e o enfermeiro deve incorporar demandas dessa especialidade. Dentre estas, destaca-se o diagnóstico de enfermagem, objeto deste estudo, que teve como objetivo comparar os diagnósticos de enfermagem, segundo Horta, de pacientes que sofreram transplante de fígado decorrente de cirrose etílica com os de cirrose não-etílica. Trata-se de um estudo retrospectivo, quantitativo, realizado em um hospital universitário de Belo Horizonte. Foram analisados 37 históricos de enfermagem de pacientes transplantados no período de primeiro de setembro de 2005 a 30 de setembro de 2006. Destes, 8 (21,6\%) eram pacientes portadores de cirrose etílica, e os demais (29-78,4\%), de nãoetílica. Foram identificadas 25 necessidades afetadas: psicobiológicas (83,6\%), psicossociais (12,7\%) e psicoespirituais $(3,7 \%)$. Ficou evidente que as necessidades psicobiológicas e espirituais não diferem entre os dois grupos. Porém, as necessidades de auto-imagem e autoconceito foram identificadas somente nos pacientes portadores de cirrose etílica.

Palavras-chave: Cirrose Hepática. Transplante de Fígado. Teoria de Enfermagem.

\begin{abstract}
The transplant, in some areas is being considered a healing option and the nurse must incorporate demands of this specialty. Amongst these, he is distinguished I diagnosis of nursing, object of this study, that had as objective to compare the nursing diagnostic, according to Horta, of decurrent transplant patients of liver of etílic cirrhosis with other cause of cirrhosis. This retrospective study with quantitative carried out in a university hospital of Belo Horizonte, Minas Gerais, Brazil. Thirty and seven descriptions of transplant patients of liver in the first period of September $1^{\text {st }}, 2005$ to September $30^{\text {th }}, 2006$ were considered for inclusion in the study. Of these $8(21,6 \%)$ were carrying of etílic cirrhosis and $29(78,4 \%)$ of not etílic cirrhosis. 25 affected necessities: psychobiology $(83,6 \%)$, psycho-social $(12,7 \%)$ and psycho-spiritual had been identified $(3,7 \%)$. It was evident that the psychobiology and psycho-spiritual necessities, from general way, do not differ between the two groups. However, the necessities of auto image and auto concept had been evident in the carrying patients of etílic cirrhosis.
\end{abstract}

Keywords:

Liver cirrhosis. Liver Transplantation. Nursing Theory.

\section{Resumen}

Los transplantes en algunas zonas pasó a considerarse una opción curativa y el enfermero está incluido en las demandas de esta especialidad. Entre estas especialidades tenemos el diagnóstico de enfermería, tema de este estudio, que tiene como objetivo equiparar los diagnósticos de enfermería, según Horta, de pacientes portadores de Cirrosis alcohólica con los de Cirrosis no alcohólica. Es un estudio retrospectivo con abordaje cuantitativo realizado en un Hospital Universitario de Belo Horizonte-MG. Para tal, fueron analizados 37 registros de enfermería referentes a pacientes transplantados de hígado con fecha de primero de septiembre 2005 hasta 30 de septiembre de 2006, con los siguientes resultados: $8(21.62 \%)$ eran pacientes con Cirrosis alcohólica y los demás $29(79.38 \%)$ con Cirrosis no alcohólica. Fueron detectadas 25 necesidades, diferenciadas en: psicobiológicas (83.6\%), psicosociales ( $12.7 \%$ ) y psicoespirituales (3.7\%). Al final del estudio, se notó que las necesidades psicobilogicas y psicoespirituales, en general, son parecidas entre los dos grupos de pacientes. Todavía, las necesidades de auto-imagen y auto-concepto fueron notadas solamente en los pacientes portadores de Cirrosis alcohólica.

Palabras clave:

Cirrosis Hepatica. Transplante de Hígado. Teoría de Enfermería. 


\section{INTRODUÇÃO}

0 desenvolvimento da tecnologia de monitorização trans e pós-operatória e a melhoria dos métodos de preservação de enxertos de qualidade e da farmacologia (imunossupressores) constituem fatores responsáveis pelo sucesso dos transplantes. A partir da década de 80 do século passado, os transplantes passaram a ser considerados opção terapêutica e curativa para muitas doenças antes consideradas terminais ${ }^{1,2,3}$.

Centros de referência e excelência de vários países atualmente realizam com sucesso transplantes de órgãos e tecidos, tais como: fígado, pâncreas, intestinos, coração, pulmão, medula óssea, córnea, ossos, tecidos músculo esquelético e cutâneo.

0 primeiro transplante hepático do mundo foi realizado em 1963 por Thomas Starzl, em Denver, nos Estados Unidos (EUA). No Brasil, em 1968, esse procedimento foi realizado pela primeira vez no Hospital das Clínicas da Faculdade de Medicina da Universidade de São Paulo (HC-FM USP) e, em Minas Gerais, no Hospital Felício Rocho em outubro de 1989. 0 Hospital das Clínicas da Universidade Federal de Minas Gerais (HC-UFMG), em setembro de 1994, iniciou seu programa de transplante hepático'.

Considerando a complexidade do procedimento cirúrgico e a necessidade de readaptação familiar e à vida sócio-laborativa pós-transplante, o paciente demanda uma assistência multiprofissional, em particular de enfermagem, por ser esta uma assistência direta e continua.

Ao repensar a assistência de enfermagem para esses pacientes, dentro de uma visão holística do ser humano, que reflita um compromisso com a excelência no atendimento, é vital que se utilizem instrumentos metodológicos do cuidar. A preocupação em desenvolver um modo de fazer, fundamentado em algum modo de pensar, levou pesquisadores, estudiosos e trabalhadores a desenvolver, desde os anos de 1950, o Processo de Enfermagem (PE) que organiza o cuidado ${ }^{4}$.

0 Processo de Enfermagem, segundo Horta ${ }^{5}$, é constituído por fases inter-relacionadas e visa à assistência ao ser humano através de ações sistematizadas. A primeira fase é o Histórico de Enfermagem, que, através de um roteiro sistematizado, permite o levantamento de dados do ser humano que são significativos para o enfermeiro. A análise destes dados possibilita a identificação das necessidades que precisam de atendimento, constituindo assim a fase de Diagnóstico de Enfermagem. A partir deste diagnóstico, elabora-se um Plano Assistencial (plano global de assistência). A implementação desse plano pelo roteiro diário, de acordo com as prioridades de cuidados, constitui a Prescrição de Enfermagem. A Evolução de Enfermagem refere-se às respostas do ser humano à assistência de enfermagem prestada. Finalmente, a estimativa da capacidade do ser humano em atender suas próprias necessidades básicas compõe a fase do Prognóstico de Enfermagem.

A primeira fase deste modelo de PE foi implementada para os pacientes do transplante hepático no $\mathrm{HC} / \mathrm{UFMG}^{6} \mathrm{em}$ setembro de 2005, fundamentada na Teoria das Necessidades Humanas Básicas (NHB) ${ }^{5}$.

Salviano ${ }^{6}$, em seu estudo com os 43 pacientes submetidos a transplante hepático no HC/UFMG no período de março de
2005 a setembro de 2006, identificou as doenças de base (Tabela 1) que os levaram ao transplante de fígado.

\section{Tabela 1:}

Distribuição dos pacientes transplantados de fígado no HC-UFMG, segundo a doença de base. Belo Horizonte 2005-2006.

\begin{tabular}{lcc}
\hline \multicolumn{1}{c}{ Doenças de base } & N & \% \\
\hline Cirrose vírus C & 8 & 18,6 \\
Cirrose vírus C e hepatocarcinoma & 8 & 18,6 \\
Cirrose criptogênica & 7 & 16,3 \\
Cirrose etanólica & 5 & 11,6 \\
Cirrose auto-imune & 3 & 7,0 \\
Cirrose biliar primária & 3 & 7,0 \\
Cirrose etanólica + vírus C & 3 & 7,0 \\
Cirrose medicamentosa & 1 & 2,3 \\
Glicogenose tipo I & 1 & 2,3 \\
Colangiopatia auto-imune & 1 & 2,3 \\
Hemocromatose & 1 & 2,3 \\
Colangite esclerosante primária & 1 & 2,3 \\
Metástase hepática Tu carcinóide & 1 & 2,3 \\
\hline
\end{tabular}

Fonte: Prontuários dos pacientes pesquisados

Pelos dados apresentados, observou-se que a maioria (38) dos pacientes transplantados era portadora de cirrose, dentre estas a etilica. Diante destes achados, fez-se a seguinte indagação: 0 perfil demográfico e as necessidades básicas de pacientes portadores de cirrose etilica diferem dos pacientes portadores de outros tipos de cirrose? Para responder a esta indagação, foi realizado este trabalho com o objetivo de comparar o perfil demográfico e necessidades humanas básicas, segundo Horta ${ }^{5}$, apresentados pelos pacientes portadores de cirrose etilica com os de cirrose não-etilica, submetidos a transplante hepático.

\section{MATERIAL E MÉTODOS}

Trata-se de um estudo descritivo, quantitativo, exploratório e retrospectivo, desenvolvido na Unidade de Transplantes de um hospital de ensino, de grande porte, da cidade de Belo Horizonte, Minas Gerais. A população do estudo foi constituída pelos Históricos de Enfermagem dos 38 pacientes transplantados de fígado no HCUFMG, em decorrência de cirrose, no período de $1^{\circ}$ de setembro de 2005 a 30 de setembro de 2006. Ressalta-se que um desses pacientes foi excluído do estudo devido à escassez de dados em seu Histórico de Enfermagem. Os dados foram transcritos para um formulário e classificados segundo a Teoria das Necessidades Básicas de Wanda de Aguiar Horta ${ }^{5}$. 0 projeto da pesquisa foi aprovado pelo Comitê de Ética em Pesquisa da UFMG (parecer nº. ETIC 0115/06), e também foram observados os princíios que regem as pesquisas que envolvem seres humanos previstos na Resolução 196/1996 do Conselho Nacional de Saúde7.

\section{CONSIDERAÇÕES GERAIS SOBRE O TRANSPLANTE HEPÁTICO}

0 candidato ao transplante hepático é submetido a uma avaliação rigorosa no período da indicação e do preparo para a 
intervenção para se definir se ele será capaz de sobreviver ao trans e pós-operatório imediato e se possui condições psicossociais adequadas para submeter-se a uma rigorosa terapêutica medicamentosa e ao acompanhamento ambulatorial prolongado no pós-transplante ${ }^{8,9,10,11}$.

As indicações de transplante hepático, segundo autores ${ }^{9,10}$ são classicamente subdivididas em 5 grupos, como apresentado.

- Doenças colestáticas: atresia de vias biliares, cirrose biliar primária e secundária, colangite esclerosante;

- Doenças da insuficiência hepatocelular: cirroses (auto-imune, pelo vírus B, C, D, alcoólica, medicamentosa, criptogenética);

- Doenças metabólicas: hemocromatose, tirosinemia, glicogenoses, deficiência de alfa-1 antitripsina, doença de Wilson;

- Vasculares: síndrome de Budd-Chiari, doença venooclusiva;

- Neoplasias: carcinoma hepatocelular (CHC) e tumores metastáticos carcinóides.

Para os mesmos autores, as contra-indicações absolutas ao transplante hepático incluem as condições psicossociais: uso de álcool em menos de seis meses; uso de drogas ilícitas em menos de seis meses por pacientes com história de drogadição; doença cardiopulmonar avançada; inabilidade de seguir o esquema de imunossupressão; tumor maligno extrahepático; septicemia incontrolável e evidência de doença neurológica grave irreversível ${ }^{9,10}$.

Como contra-indicações relativas têm-se a idade; cirurgias abdominais prévias; desnutrição grave; obesidade grave; insuficiência renal (creatinina $>2 \mathrm{mg} / \mathrm{dl}$ ); tumores hepatobiliares; trombose da veia porta e infecção pelo vírus da imunodeficiência adquirida ${ }^{8,11}$.

No hospital, campo do estudo, após ser submetido ao transplante, o paciente é transferido rotineiramente para o CTI. Com sua estabilização hemonidâmica e ventilatória, a constatação de início da função do enxerto e na ausência de complicações cirúrgicas agudas, o paciente obtém alta do CTI e é encaminhado à Unidade de Transplante; o período de permanência nessa Unidade é em torno de duas semanas.

0 enfoque assistencial multiprofissional ao paciente internado na Unidade de Transplantes nesse período envolve o ajuste do esquema imunossupressor, monitorização dos parâmetros da função hepática, renal, hematológica, bioquímica do sangue, o rastreamento rigoroso de possíveis sinais de infecção e o acompanhamento do estado emocional e afetivo do paciente bem como de suas condições sociais e econômicas para adequar-se ao novo ritmo de vida após a alta hospitalar.

Nessa fase, o enfermeiro assistencial deve estar alerta para os diagnósticos e intervenções de enfermagem, de modo a alcançar os resultados esperados concernentes ao atendimento às necessidades psicobiológicas, psicossociais e psicoespirituais. A educação em saúde, nesse período, deve ser então enfatizada, com abordagem multidisciplinar.

0 paciente é submetido a um plano de fisioterapia motora e respiratória, mediante demanda específica, e fica a cargo da psicologia o suporte emocional e psicoterápico para o paciente, na adaptação com o novo órgão e o preparo para reestruturação da vida sócio-familiar. Além disso, o paciente conta com assistência social quando não possui recursos para arcar com as despesas inerentes à sua nova condição de vida após a alta hospitalar.

\section{RESULTADOS E DISCUSSÃO}

0 estudo foi desenvolvido utilizando dados prontuários de 37 pacientes em pós-operatório de transplante decorrente de cirrose hepática. Destes, 8 (21,6\%) por cirrose etílica (grupo etílico) e $29(78,4 \%)$ por outras causas (grupo não-etílico).

A maioria dos pacientes portadores de cirrose era do sexo masculino, sendo que os portadores de cirrose etílica eram todos desse gênero.

De acordo com a literatura, o maior consumo abusivo de álcool está entre os homens. Um estudo chinês ${ }^{12}$ mostrou prevalências de alcoolismo de $16 \%$ para os homens e $2 \%$ para as mulheres. Outro, com pacientes hospitalares brasileiros ${ }^{13}$, detectou uma prevalência de $22 \%$ entre os homens e apenas $3 \%$ entre as mulheres. Logo, o fato de a totalidade dos pacientes portadores de cirrose etílica ser do sexo masculino condiz com os dados estimados pela literatura em relação ao consumo abusivo de bebidas alcoólicas por pessoas desse sexo.

Dentre as doenças hepáticas, a cirrose etílica é a principal causa de morbimortalidade. A lesão hepática está diretamente relacionada ao volume e tempo de consumo e teor alcoólico da bebida. Estudos mostram que cerca de $20,0 \%$ dos portadores de cirrose etílica consumiram cerca de $160 \mathrm{~g} /$ dia por 8 a 10 anos de algum tipo de bebida alcoólica ${ }^{9,10}$.

0 conhecimento do estado civil do paciente é muito importante para o enfermeiro, pois este deve envolver a família no cuidado do paciente no pós-operatório. Todos os pacientes do grupo "cirrose etílica" eram casados. Este fato é importante, pois, pelo menos teoricamente, o paciente conta com um vínculo familiar.

Quanto à escolaridade, não se evidenciou diferença entre os dois grupos. Em ambos, cerca de $50 \%$ dos pacientes têm nível médio ou superior e exercem atividades autônomas.

Na Tabela 2, a seguir, estão discriminadas as NHB afetadas sobre as quais foram encontrados registros.

São comentadas apenas as necessidades que se manifestaram, no mínimo, por $40 \%$ dos pacientes. Na população estudada, a totalidade dos pacientes, dos dois grupos, apresenta dificuldade para locomoção e para qualquer tipo de atividade física. Conseqüentemente, apresentam incapacidade para autocuidado em graus diferenciados.

Os fatores relacionados a essa síndrome, encontrados na população estudada, são corroborados pela literatura e se referem aos relativos à doença de base crônica debilitante, sendo esta, neste caso, a falência hepática; ao tratamento pósoperatório de cirurgia de grande porte que causa fadiga e dor; e às restrições emocionais e ambientais.

Os pacientes do grupo "cirrose etílica" encontravam-se mais debilitados que os demais, em decorrência da má nutrição pela inapetência que é comum nos alcoólatras e, posteriormente, pela disfunção hepática. Esta condição é agravada pelo desconfor to e dor inerente ao pós-operatório imediato de cirurgia de grande 
porte e aos prejuízos músculo-esqueléticos ocasionados pela diminuição da força e da massa muscular. Conseqüentemente, esses pacientes eram mais dependentes em relação aos demais, no que se refere às atividades que exigiam esforço físico e deambulação, e ainda apresentavam certo grau de diminuição ou prejuízo sensório-perceptivo e cognitivo, no pós-operatório.

Tabela 2:

Necessidades humanas básicas afetadas apresentadas por pacientes submetidos a transplante hepático, segundo o tipo de cirrose. Belo Horizonte, 2005-2006.

\begin{tabular}{|c|c|c|}
\hline NHB afetadas & Cirrose etílica Cirrose & não- etílica \\
\hline \multicolumn{3}{|l|}{ Psicobiológicas } \\
\hline Oxigenação & $x$ & $x$ \\
\hline Eliminação & $x$ & $x$ \\
\hline Nutrição & $x$ & $x$ \\
\hline Cuidado corporal & $x$ & $x$ \\
\hline Hidratação & $x$ & $x$ \\
\hline Locomoção & $x$ & $x$ \\
\hline Exercício e Atividades físicas & $x$ & $x$ \\
\hline Integridade cutâneo-mucosa & $x$ & $x$ \\
\hline Regulação térmica & $x$ & $x$ \\
\hline Percepção dolorosa & $x$ & $x$ \\
\hline Regulação imunológica & $x$ & $x$ \\
\hline Percepção visual & $x$ & $x$ \\
\hline Percepção tátil & $x$ & $x$ \\
\hline Percepção auditiva & $x$ & $x$ \\
\hline Segurança física & $x$ & $x$ \\
\hline Sono e Repouso & $X$ & $x$ \\
\hline Ambiente & $x$ & $x$ \\
\hline Sexualidade & $x$ & $x$ \\
\hline \multicolumn{3}{|l|}{ Psicossociais } \\
\hline Aprendizagem / Educação à saúde & $x$ & $x$ \\
\hline Segurança emocional & $x$ & $x$ \\
\hline Comunicação & $x$ & $x$ \\
\hline Orientação no tempo e no espaço & $x$ & \\
\hline Auto-imagem & $x$ & \\
\hline Auto-estima & $x$ & \\
\hline \multicolumn{3}{|l|}{ Psicoespiritual } \\
\hline Religiosa & $x$ & $x$ \\
\hline
\end{tabular}

A regulação imunológica é uma necessidade de todos os pacientes submetidos a transplante devido ao uso de imunossupressores, independente da doença de base. Esta condição é acentuada pelo estado nutricional do paciente. Portanto, toda equipe de saúde deve enfatizar a prevenção e o controle de infecção em pacientes imunossuprimidos.

0 desequilíbrio térmico pode ser apresentado por todos os pacientes em pós-operatório. No caso de transplante, a elevação da temperatura pode estar relacionada a processos de rejeição aguda ${ }^{14}$. Esse sintoma pode ainda estar decorrente do uso de alguns imunossupressores como o tacrolimus e as imunoglobulinas $^{15}$. A hipertermia foi apresentada apenas por 6 dos pacientes. Os demais possuíam fatores de risco para 0 desequilíbrio da temperatura corporal, tais como alteração da taxa metabólica, medicações, inatividade, idade, peso e desidratação.

Em pacientes com disfunção hepática grave, como as que exigem transplante para sobrevivência do paciente, comumente aparecem as aranhas vasculares decorrentes da hipertensão portal, as hemorragias digestivas devidas à erosão da mucosa esofagogástrica, a ginecomastia em homens, prurido generalizado, icterícia, edema de extremidades, podendo evoluir para anasarca, e desnutrição seletiva por alterações metabólicas e de absorção ${ }^{10}$.

Todas essas condições prejudicam o tecido cutâneo, subcutâneo e mucosas e são exacerbadas pela intervenção cirúrgica de grande porte, pela pressão em proeminências ósseas, contato com secreção e excreções corporais, uso de imunossupressores e dificuldade de mobilidade física. Esta condição é apresentada por todos os pacientes, independente da causa da cirrose.

As alterações do estado mental ocorrem em $10 \%$ a $30,0 \%$ dos pacientes de transplante hepático ${ }^{14}$. Na maioria dos casos, podem se relacionar, entre outros fatores, com a privação do sono e desorientação da psicose da terapia intensiva. Esses eventos podem se manifestar na Unidade de Internação, o que ocorreu com todos os pacientes do grupo "cirrose etílica" que apresentaram alterações na orientação tempo-espaço.

A recuperação das funções endócrinas após o transplante hepático é confirmada por Strouse ${ }^{16}$. No que se refere à sexualidade, embora haja um despertar dessas funções no pós-operatório, outros fatores afetam a percepção do paciente quanto a sua condição para o seu desempenho sexual. Dentre estes fatores, estão a depressão pós-operatória, que pode ocorrer, alterações da auto-imagem e auto-estima, experiências e relacionamentos anteriores.

Diante de um pós-operatório satisfatório, a grande expectativa dos pacientes está voltada para sua sexualidade. Neste aspecto, percebe-se que os pacientes do grupo "cirrose etílica" ficam mais ansiosos e receosos, pois esta necessidade pode estar afetada não só pela cirrose em si, mas previamente pelo alcoolismo.

A diarréia é uma condição clínica comum após o transplante hepático, o que gera um grande impacto na assistência de enfermagem, uma vez que muitos pacientes têm incontinência fecal associada à diarréia. Esta condição associada a maior debilidade física e alterações do estado mental, como encontrado em pacientes de cirrose etílica, leva o paciente a um maior grau de dependência.

A compreensão sobre sua condição de saúde e adesão ao tratamento constitui pré-requisitos básicos para inclusão do paciente no programa de transplante. No entanto, embora 0 enfermeiro tenha orientado o paciente e a família, no préoperatório, sobre toda a trajetória do transplante, ao se aproximar o dia da alta hospitalar, surgem muitas dúvidas mediante o receio de voltar para casa e o sentimento de impotência para o autocuidado. Este problema foi encontrado em $100 \%$ dos pacientes envolvidos no estudo.

A ansiedade é o sentimento de intranqüilidade e apreensão que surge em resposta a uma ameaça inespecífica. A ansiedade se diferencia do medo apenas pela ausência de ameaça ${ }^{17}$. Na prática clínica, ambos os sentimentos podem coexistir e produzem a mesma resposta simpática, como alteração da freqüência cardíaca, dilatação das pupilas, sudorese, tremores e secura na boca.

A necessidade religiosa ou espiritual foi pouco identificada ou registrada para ambos os grupos. Pode-se supor que ou os pacientes realmente não manifestaram essa necessidade de assistência ou esta não foi percebida pelos elementos da equipe de enfermagem. Outra hipótese ainda é de que essa necessidade foi percebida e foi prestada a assistência necessária para cada caso, porém não foi registrada, como pode acontecer na nossa pratica diária. 
A necessidade espiritual manifestada pelos pacientes pode ser um meio de superação do sofrimento e de aquisição de forças para resistir à dor física do pós operatório, à angustia e à ansiedade mediante uma nova oportunidade de vida decorrente da morte de outra pessoa.

Assim, concordamos com Pietrukowicz ${ }^{18}$ quando diz que "a fé é o alimento que dá à vida esperanças de dias melhores e ajuda a enfrentar as experiências mais dramáticas do cotidiano".

As necessidades psicossociais de orientação no tempo e espaço, auto-imagem e autoconceito só foram detectadas, ou no mínimo só registradas, para os pacientes do grupo "cirrose etilica".

Os distúrbios de orientação no tempo e no espaço podem ser decorrentes da terapêutica medicamentosa, especificamente de analgésicos e sedativos utilizados usualmente no pós-operatório de cirurgia de grande porte e no caso do paciente de cirrose etílica, agravados pelas condições psíquicas preexistentes.

As alterações de auto-imagem e autoconceito podem estar relacionadas à condição física atual pós-transplante, caracterizada pela debilidade física e dependência de outros para suprir suas atividades de vida diária (AVD), agravada por sentimentos ligados ao estilo de vida prévio (alcoolismo).

0 ex-alcoólatra, como é o caso de paciente transplantado de fígado por cirrose etílica, traz representações que perpassam a dimensão física, social, mental e espiritual. Portanto, envolvendo o indivíduo em sua totalidade. 0 uso abusivo do álcool afeta não só o físico, mas também deteriora as relações sociais, notadamente na família e no trabalho, levando a alterações psicossociais com repercussões na auto-imagem e no autoconceito ${ }^{19}$.

0 uso indiscriminado de bebida alcoólica, droga psicoativa, como é o caso dos pacientes portadores de cirrose etílica, pode promover alterações físicas e comportamentais no indivíduo ${ }^{20}$.

As alteraçõess comportamentais afetam, entre outras, as necessidades psicossociais que, segundo Mohana ${ }^{21}$, podem ser definidas como tendência que todo ser humano tem de conversar, conviver socialmente, firmar-se perante os outros, tornar visível seus valores, sentir-se querido, amado e aceito pelo outro. Dentre estas necessidades, destacamos as de auto- imagem e autoconceito.

Portanto, pode-se inferir que as manifestações das necessidades de auto-imagem e autoconceito por parte dos pacientes transplantados de fígado por cirrose etílica podem estar ligadas não só pela questão da doença e tratamento em si, mas pela condição do alcoolismo.

Partindo-se do pressuposto de que os pacientes que são submetidos à cirurgia de transplante hepático em decorrência de cirrose etílica fizeram uso abusivo de bebidas alcoólicas, pode-se supor que as necessidades de auto-imagem e autoconceito estejam afetadas não só em decorrência da

\section{Referências}

1. Garcia VD. História dos transplantes no Brasil.[on line] 2002. Disponível em: http://www.abto.com.br. debilidade física, mas também de comportamentos decorrentes do alcoolismo. Assim, estes pacientes poderão ter mais dificuldades para retomar uma "nova vida" pós- transplante, uma vez que sua auto-imagem e seu autoconceito estão diminuídos, seja pelo sentimento de culpa ao lembrar-se de suas relações familiares e sociais e também pelas debilidades decorrentes do alcoolismo, da cirrose hepática e, ainda, de uma cirurgia de grande porte. Portanto, a assistência da equipe deve estar também voltada para elevar o autoconceito e auto-imagem, fundamentais para sua recuperação e reabilitação física, psíquica e social.

\section{CONSIDERAÇõES FINAIS}

Em síntese, pode-se dizer que, em relação às necessidades biológicas, não houve diferença entre os pacientes transplantados de fígado por cirrose etílica e os transplantados por cirrose de outras causas. Os pacientes de ambos os grupos apresentaram as mesmas necessidades, porém em graus diferenciados. Observou-se que pacientes do primeiro grupo eram mais debilitados e, portanto, mais dependentes da equipe de enfermagem que os demais pacientes.

Quanto às necessidades psicobiológicas, a auto-imagem, o autoconceito e a orientação no tempo e no espaço, no pós-operatório na Unidade de Internação, só foram manifestados pelos pacientes que tinham cirrose etílica, provavelmente em decorrência do estilo de vida prévia, com repercussões significativas na área psicossocial.

A manifestação da necessidade espiritual, embora pouco registrada, foi semelhante nos dois grupos. Isto pode indicar que não importam as causas que levaram a um transplante, mas sim o fato de que as pessoas, de ambos os grupos, estavam na mesma condição de pós-operatório, buscando na espiritualidade conforto e esperança de uma recuperação sem complicações.

A chave do sucesso dos resultados após a alta hospitalar é a educação do paciente ${ }^{22}$. Ao assistir aos pacientes de transplante, torna-se evidente a importância da família como unidade do cuidado, pois nela o paciente se apóia para enfrentamento de seus desafios de saúde ${ }^{23}$. Portanto, essa força deve ser potencializada pelo enfermeiro, no seu papel educativo, durante a permanência do paciente na Unidade de Internação.

Uma das grandes preocupações da equipe de saúde, em especial da Enfermagem, por estar vinculada à educação do paciente e da família, refere-se aos pacientes transplantados em decorrência de cirrose etílica. Sabe-se que o alcoolismo é uma doença que apenas é controlada, e, portanto, há risco de recidiva em relação ao uso de bebida alcoólica. Assim, o suporte da família e da comunidade deve ser otimizado para ajudar o paciente a ter um estilo de vida saudável e administrar ou controlar as tarefas adaptativas relacionadas aos novos desafios que terá de enfrentar.

2. Duarte MMF, Salviano MEM, Gresta MM. Assistência de enfermagem. In: Pereira WA. Manual de transplantes de órgãos e tecidos. $3^{\mathrm{a}}$ ed. Rio de Janeiro (RJ): Medsi; 2004.

3. Starzl T. Back to the future. Transplantation 2005 May; 79 (9): 1009-14. 
4. Garcia TR, Nóbrega MML, organizadores. Processo de enfermagem e os sistemas de classificação dos elementos da prática profissional. São Paulo: (SP): Atheneu; 2004.

5. Horta WA. Processo de enfermagem. São Paulo (SP): Edusp; 1979.

6. Salviano MEM. Transplante hepático: diagnósticos de enfermagem segundo a Nanda em pacientes no pós-operatório na unidade de internação [dissertação de mestrado] Belo Horizonte (MG): Escola de Enfermagem /UFMG; 2007.

7. Conselho Nacional de Saúde. Resolução CNS n ${ }^{0} 196$ de 10 de outubro de 1996. Aprova as diretrizes enormas regulamentadoras de pesquisa envolvendo seres humanos. Inf Epidemiol SUS 1996 abr/jun; 5 (2 supl 3): 13-41.

8.Raia SMA, Mies S. Transplante de fígado. In: Raia AA, Zerbini EJ. Clínica cirúrgica. $4^{a}$ ed. São Paulo (SP): Sarvier; 1988.

9. Castro LP, Coelho LGV. Gastroenterologia. Rio de Janeiro (R): Medsi; 2004.

10. Sherlock S, Dooley J. Doenças do fígado e do sistema biliar. $11^{\mathrm{a}}$ ed. Rio de Janeiro (RJ): Guanabara Koogan; 2004.

11. Busuttil RW, Klintmalm GB. Transplantation of the liver. $2^{\text {nd }} e d$. Philadelphi(USA): Elsevier Saunders; 2005.

12. Khan N, Davis P, Wilkinson TJ, Sellman JD, Graham P. Drinking patterns among older people in the community: hidden from medical attention? New Zeland Med J 2002;115: 72-75.

13. Figlie NB, Pillon SC, Dunn J, Laranjeira R. The frequency of smoking and problem driking among general hospital inpatients in Brasil-using the AUDIT and Fagerstrom questionnaires. São Paulo Med J 2000;1 18(5):139-43
14.Everson GT, Kam I. Immediate postoperative care. In: Madderey WC, Sorrel MF, Schiff ER. Transplantation of de liver. 3nd ed. Philadelphia.(USA) Lippincott Willians \& Wilkins; 2001.

15. Clavien P, Killenberg PG. Medical care of the liver transplantation patient. Ontario: Blackwell Science; 1997.

16. Strouse TB. Neuropsychiatric outcomes in liver transplant recipients. In: Busuttil RW, Klintmalm GB. Transplantation of the liver. $2^{\text {nd }}$ ed. Philadelphia(USA): Elsevier Saunders; 2005.

17. Carpenito LJ. Diagnósticos de enfermagem: aplicação à prática clínica. 10 ed. Porto Alegre (RS). Artmed; 2005.

18. Pietrukowicz MCLC. Apoio social e religião: uma forma de enfrentamento dos problemas de saúde. [dissertação de mestrado]. Rio de Janeiro (RJ): Escola Nacional de Saúde Pública/ FIOCRUZ; 2001.

19. Campos EA. Representations of alcoholism in a former alcoholics Association Acoholics Anonymous. Cad Saude Publica, 2004; 20(5)

20. Noto AR, Carlini EAA. Internações hospitalares provocadas por drogas: análise de sete anos consecutivos (1987-1993). Rev ABP-APAL; 1995.

21. Mohama J. 0 mundo e eu. Rio de Janeiro(RJ): Agir; 1963.

22. Kunder G. Role of the posttransplant coordinator. In: Busuttil RW, Klintmalt GB. Transplantation of the liver. 2nd ed. Philadelphia(USA): Eselvier Saunders; 2005.

23. Wright LM, Leahey M. Avaliação e intervenção na família. Tradução de Sílvia M. Spada. $3^{\text {a }}$ ed. São Paulo (SP): Roca; 2002. 